\title{
Consideration of the normality of experimental data
}

GERALD H. FISHER

UNIVERSITY OF NEWCASTLE UPON TYNE, ENGLAND

Many experimental studies are designed in such a way as to allow statistical consideration of the normality of emergent data. This entails determination of the frequencies expected to be reflected in a standardised normal deviate apportioned to an appropriate number of categories. $A$ new method of tabulating percentage frequencies expected to fall in normally distributed samples of data is illustrated.

In a previous paper, Fisher (1967) drew attention to certain difficulties experienced in extracting data from statistical tables cast in conventional form. In this he illustrated a new method for presenting data referring to probability values associated with the standard normal distribution function. The principal advantage of this method of presentation lies in the fact that data can be obtained from it very much more quickly than from the usual matrix-type table, fewer errors being made in the course of so doing.

Of the order of $80 \%$ of statistical procedures in common use proceed from the assumption that the data under consideration have been drawn from populations of events which are normally distributed. All so-called "parametric" statistics explicitly or implicitly involve an assumption of this kind. Many experiments are designed in such a way as to allow this assumption to be questioned, and frequently statistical consideration of the essential "normality" of emergent data is a prerequisite either for further analysis or for more extensive experimentation.

The mathematical procedures involved in determining whether or not data may be considered to have been drawn from populations, the parameters of which are known, or which may be defined, are referred to as "curve-fitting." In general, they entail evaluation of the probability of the outcome of a particular experiment, expressed in numerical form, being commensurate with a specified expectation. This is usually expressed in terms of a formal statement referred to as a "prediction." The extent to which the results obtained in the experiment differ from those expected on the relevant hypothesis is computed in an appropriate way and the outcome evaluated accordingly.

When either the prediction or assumption of normality is under consideration, expected frequencies may be obtained from a table, the entries in which refer to the probabilities associated with standardized normal deviates. First, the number of categories relevant to the particular distribution of data is decided upon according to the form in which it has been collected, or on some other appropriate criterion. Secondly, if the data have become available in the form of a cumulative distribution of frequen- cies, as for example in the case of a threshold-type experiment, it is necessary to uncumulate them. Thirdly, the frequencies expected to occur must be obtained. To calculate expected frequencies one is usually involved in a lengthy series of exercises, extracting information from tables which subsequently requires reorganization so as to yield estimates of the percentages falling within appropriate areas. Finally, the specific frequencies expected to appear throughout the data are calculated according to the number of cases involved in the original sample of results. One is then in a position to embark upon an appropriate curve-fitting procedure.

As in the case of extracting probability values referring to standardized normal deviates, these operations are more tedious than difficult. Here again, however, confusion arises in carrying them out and error is frequently introduced, particularly when the number of categories involved becomes relatively large.

The table reproduced in Fig. 1 is an attempt to reduce these difficulties by illustrating the percentage frequencies expected to be reflected in distributions of data drawn at random from a normal population. Appropriate percentages have been calculated from two through 13 categories, and these are entered against each distribution along with the standardized points of division of the abscissa. Hence, the table may be used directly to consider if distributions of data deviate appreciably from normality using a curve-fitting technique such as Chi square.

This is a further example of a set of statistical tables prepared by Fisher (1966), all of which are illustrated in pictorial and graphical form. I am grateful to Dr. W. Tauss of the University of Western Australia for pointing out that the data entered in this table may be cumulated, thus obviating the need for uncumulating experimental results. Dr. Tauss suggested further that the table may be used for the purpose of normalizing observed valuesthat is to say, to recast numerical values indicating the outcome of a particular experiment on the assumption that the population of events under observation is normally distributed-but care should be taken to ensure that this assumption is met by particular results before extending the use of the table in this way.

References

FISHER, G. H. The new form statistical tables. (2nd ed) London: University of London Press, 1966.

FISHER, G. H. A new method of presenting data referring to the standard normal distribution function. Psychon. Sci., 1967, 8, 455-456. 

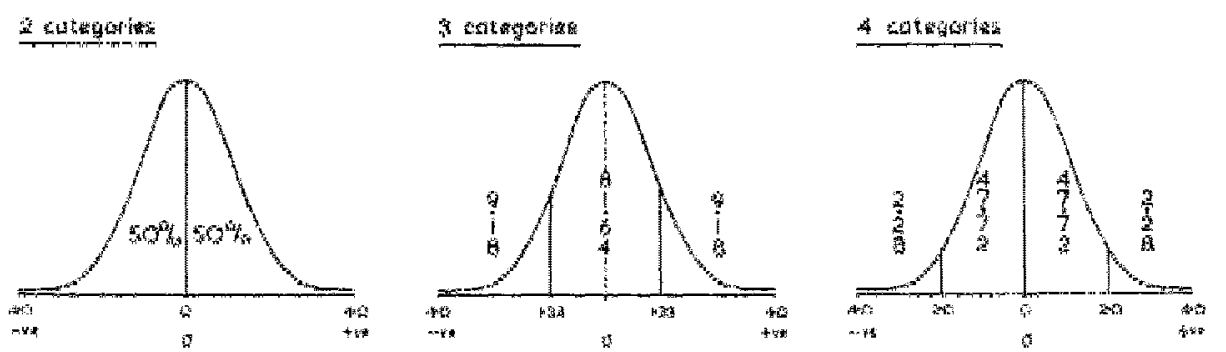

sothontas

sotedaries

7 Gategerists

13 ratiogariza
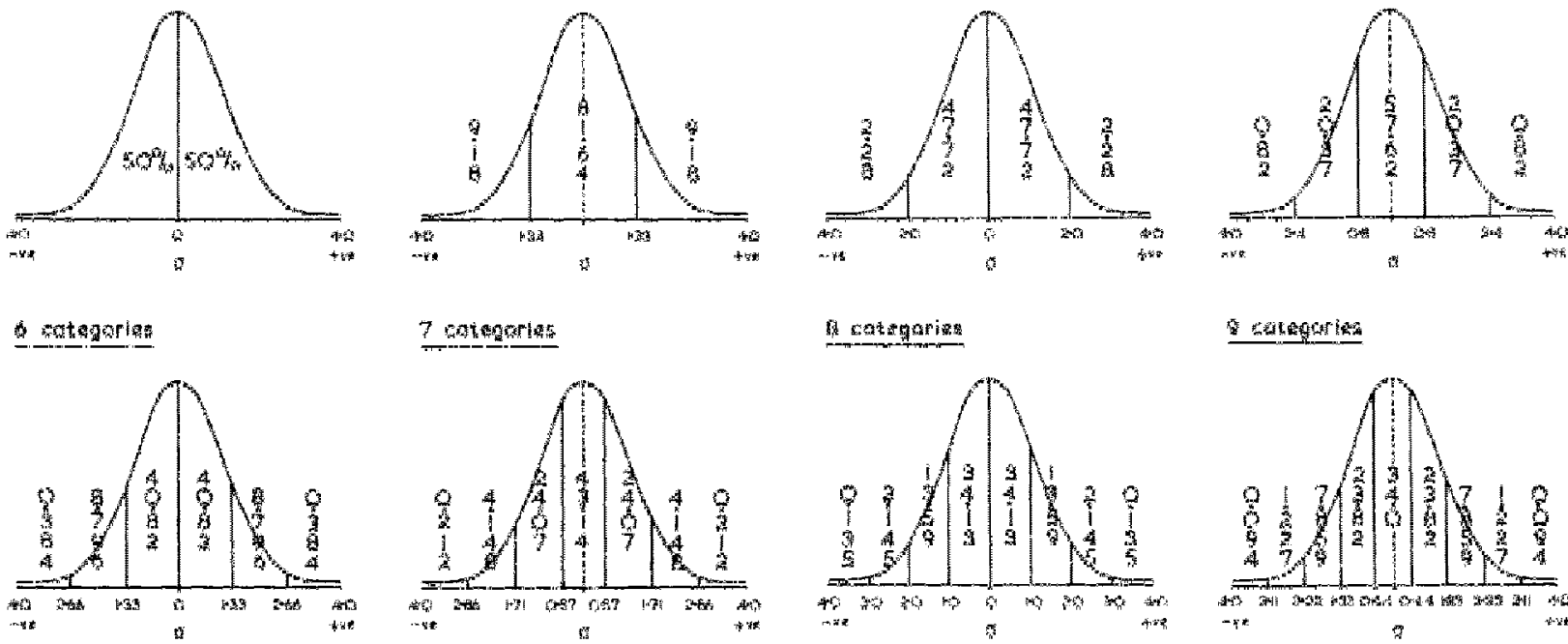

Aesteguries

10 catequrias

11 cattegariati

12 zateguries
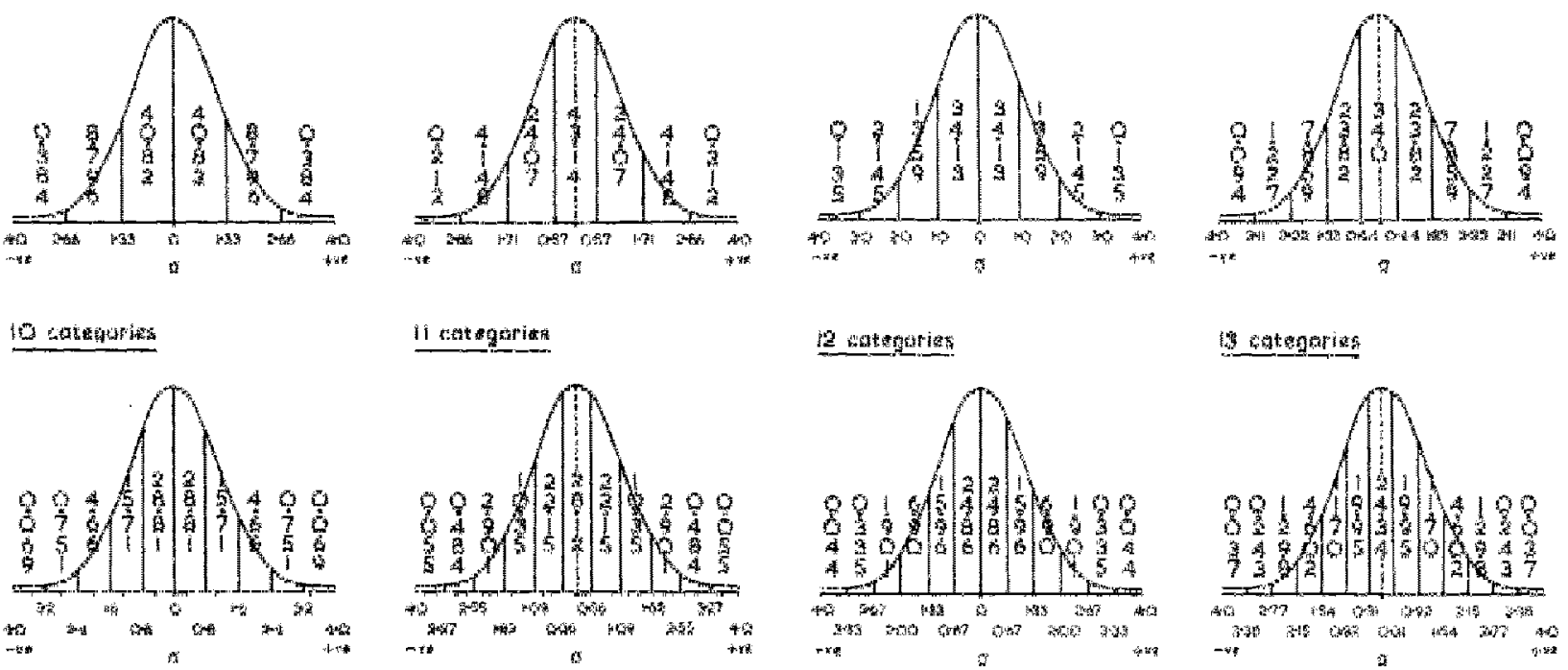

13 categariaf

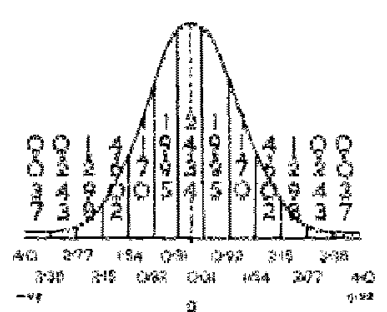

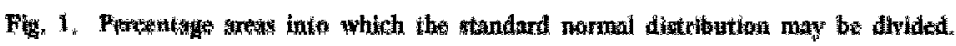

